\title{
An integral treatment of friction during a swash uprush
}

\author{
Nimish Pujara ${ }^{1 *}$ Philip L.-F. Liu ${ }^{1,2,3}$ and Harry Yeh $^{4}$
}

April 28, 2016

\author{
${ }^{1}$ School of Civil and Environmental Engineering, Cornell University, Ithaca, NY 14853, USA \\ ${ }^{2}$ Institute of Hydrological and Oceanic Sciences, National Central University, Jhongli, Taoyuan \\ 320, Taiwan \\ ${ }^{3}$ Department of Civil and Environmental Engineering, National University of Singapore, \\ Singapore \\ ${ }^{4}$ School of Civil and Construction Engineering, Oregon State University, Corvallis, OR 97331, \\ USA
}

\begin{abstract}
The effects of bed friction are modelled for the flow near the moving shoreline during the uprush phase of a swash event by extending the Pohlhausen method used by Whitham (1955) to a sloping bed. The tip of the swash near the moving shoreline is treated in an integral sense as a region of uniform velocity, being acted on by the forces of friction, gravity, and the pressure force induced by the frictionless flow behind the swash tip. The bed shear stress is parameterized by using the quadratic dependence on velocity. The theory is compared to data of the shoreline velocity and position in the swash of breaking solitary waves, and the friction coefficient is determined from direct measurements of bed shear stress. The theoretical predictions are in good agreement with the laboratory results in terms of time history of the shoreline velocity and position, as well as the run-up.
\end{abstract}

\section{Introduction}

The swash uprush is the landward movement of water after an incident wave reaches the shoreline. During this phase, the shoreline climbs the beach to reach its run-up, which is defined as the vertical distance between the highest position achieved relative to the initial position of the shoreline. Interest in the swash uprush flow stems from the fact that it is responsible for large volumes of sediment transport (e.g. review by Brocchini and Baldock, 2008) and that it is the cause of flooding due to beach overtopping (Peregrine and Williams, 2001; Baldock et al., 2012). A commonly made assumption to understand the swash flow considers the travel of a wave onto an impermeable sloping beach of initially quiescent water. As the wave climbs the beach into shallower water, the wave transforms, breaks, and reaches the shoreline where it collapses to drive the swash. The

${ }^{*}$ Corresponding author. E-mail: np277@cornell.edu. Phone: +16073794272 
swash flow is thus driven by complex non-linear processes such as growth of wave amplitude, wave breaking, and bore collapse that are still poorly understood.

For numerical solvers, the major challenges to simulating this situation are the non-linear wave transformation and bore collapse processes, as well as the three-dimensional wave breaking and wave-breaking-generated turbulence (e.g. Lin et al., 1999; Lynett et al., 2002; Zhang and Liu, 2008). The moving shoreline is also a challenge to model since it is a time-varying boundary condition to the flow (Packwood and Peregrine, 1981; Borthwick et al., 2006; Antuono et al., 2012; Pedersen et al., 2013). On the other hand, laboratory experiments face difficulties in making measurements of the same processes due to shallow flow depths, entrained air, and unsteady dynamics (Cowen et al., 2003; Jensen et al., 2003; Sou and Yeh, 2011).

One of the major unsolved challenges is the dynamics of the leading tip of the swash, i.e., the flow in the vicinity of the moving shoreline after bore collapse. It is known that flow in this region is significantly affected by the bed friction as the front propagates by continuous breaking of the water surface. Experimental data presented in Pujara et al. (2015) showed that 'the swash solution' to the inviscid shallow water equations on a sloping beach (Shen and Meyer, 1963; Peregrine and Williams, 2001) correctly predicts the flow evolution behind the moving shoreline in the swash of breaking waves, but the motion of the shoreline is not correctly predicted. Direct measurements of bed shear stress at the moving shoreline demonstrated that friction significantly afffects the flow in the swash tip leading to shoreline deceleration, and eventually to a reduced run-up. These experimental observations support the assumption made in previous studies of the closely-related dam-break flows (Whitham, 1955; Hogg and Pritchard, 2004; Ancey et al., 2008; Chanson, 2009): friction is a dominant force near the moving front where the water depth goes to zero, whereas inertia and pressure gradients dominate the dynamics away from the front.

Treatments of friction at the leading front of dam-break flows on a horizontal bed (Whitham, 1958; Hogg and Pritchard, 2004) have not yet been extended to the swash uprush despite the similarity between them (Peregrine and Williams, 2001; Pujara et al., 2015). The first aim of this paper is to extend the integral treatment of the friction-affected front of a dam-break flow on a horizontal bed (Whitham, 1955) to the friction-affected tip during a swash uprush on a sloping beach. While the matched asymptotes approach of Hogg and Pritchard (2004) shows more detailed solutions that include the variation of water depth and velocity within the friction-affected front, their leading order solution closely matches that of Whitham (1955). For the swash uprush, since detailed flow measurements in the friction-affected front are not available, we limit our attention to extending only the leading order solution to the swash uprush on a sloping bed.

The second aim this paper is to present and discuss the application of this integral treatment of friction in the swash tip during uprush to predict shoreline motion due to breaking solitary waves. We obtain predictions of shoreline motion that include the effects of bed friction and compare them to experimental data of shoreline position and shoreline velocity. Data of shoreline motion are still rare and comparisons between data and models usually only consider the shoreline position, and not the shoreline velocity. Comparison of the theory to the latter reveals the limitations of shallow water theory at very short times after bore collapse (Whitham, 1958; Yeh et al., 1989).

Previous efforts to consider the effects of friction on shoreline motion (e.g. Kirkgöz, 1981; Hughes, 1995; Puleo and Holland, 2001; Svendsen, 2006) have done so by adding a frictional term of the form $f u^{2} / h$ (with the water depth in the denominator replaced by a constant) to the equation of motion for a fluid parcel that otherwise follows a ballistic motion. However, experimental results have shown that fluid parcels do not follow a ballistic motion, but instead converge at the swash tip (Baldock et al., 2014). Further, Antuono et al. (2012) used asymptotic expansions of the nonlinear shallow water equations including a term of the form $f u^{2} / h$ in the vicinity of the shoreline 


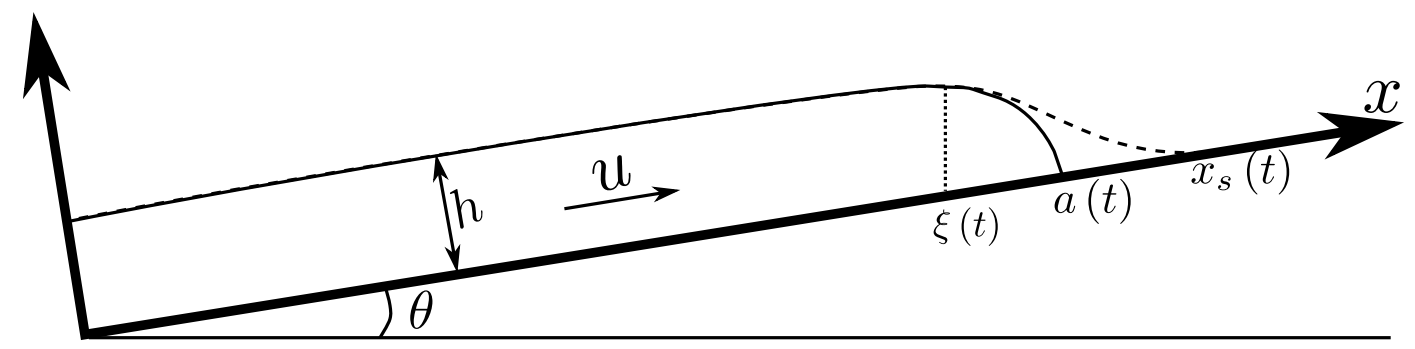

Figure 1: Definition sketch.

to show that the shoreline never recedes from its highest position. This non-physical result shows that previous solutions may rely on assumptions that have been cast into doubt. In this paper, we focus only on the uprush and provide an alternative treatment of friction on shoreline motion.

This paper is organized as follows. We show the formulation to derive the equations of motion for the swash tip in section 2, and show the solutions and comparisons to experimental data in section 3. Section 4 gives the conclusions.

\section{Formulation}

The non-linear shallow water equations (NSWE) for flow in the swash zone are written in a coordinate system parallel and perpendicular to the beach (see figure 1) as

$$
\begin{aligned}
\frac{\partial h}{\partial t}+\frac{\partial(h u)}{\partial x} & =0 \\
\frac{\partial u}{\partial t}+u \frac{\partial u}{\partial x}+g \cos \theta \frac{\partial h}{\partial x}+g \sin \theta & =0
\end{aligned}
$$

where $g$ is the gravitational acceleration, $u(x, t)$ is the depth-averaged velocity parallel to the beach, $h(x, t)$ is the water depth measured perpendicular to the beach surface, and $\theta$ is the beach slope. The NSWE are one-dimensional equations representing the conservation of mass and momentum, respectively, and are known to be a good approximation to the flow motion in the swash zone (Peregrine, 1972) except for the effects of bed friction, which is ignored in these equations. A swash event is driven by the process of bore collapse when a bore or a breaker reaches the shoreline (reviewed in Meyer and Taylor, 1972). The resulting shoreline motion is given by (Shen and Meyer, 1963)

$$
\begin{array}{r}
x_{s}=U_{s} t-\frac{1}{2} g(\sin \theta) t^{2}, \\
u_{s}=U_{s}-g(\sin \theta) t,
\end{array}
$$

where $x_{s}(t)$ is the shoreline position, $u_{s}(t)$ is the shoreline velocity, $U_{s}=u_{s}(0)$ is the initial shoreline velocity, and $t=0$ denotes the start of the swash. Peregrine and Williams (2001) provided a solution to the NSWE for the entire swash, i.e., $(x, t)>(0,0)$, by extending the asymptotic results of Shen and Meyer (1963). This 'swash solution' gives the flow evolution as 


$$
\begin{array}{r}
h(x, t)=\frac{1}{g}\left[\frac{1}{3} U_{s}-\frac{1}{6} g(\sin \theta) t-\frac{1}{3} \frac{x}{t}\right]^{2}, \\
u(x, t)=\frac{1}{3} U_{s}-\frac{2}{3} g(\sin \theta) t+\frac{2}{3} \frac{x}{t},
\end{array}
$$

which is the same as the solution to the NSWE for a dam-break flow on a sloping bed in which the initial water depth behind the dam is $U_{s}^{2} / 4 g$ (Peregrine and Williams, 2001). In this "swash solution', the shoreline climbs the beach for $g(\sin \theta) t / U_{s} \leq 1$ and its furthest position along the beach is given by $g(\sin \theta) x / U_{s}^{2}=0.5$. Antuono and Hogg (2009) and Antuono (2010) give further details of the inviscid swash flow from similar initial conditions. In the inviscid swash solution described above, the shoreline is approached with free-surface tangential to the bed, but frictional effects lead to a 'blunt nose' and flow deceleration so that the actual shoreline position, $a(t)$, is behind the frictionless shoreline solution, $x_{s}(t)$, as indicated in figure 1 . If friction with the bed only alters the dynamics in the region immediately behind the moving shoreline, which we term the 'swash tip' region, then denoting $\xi(t)$ as the interface between the friction-affected swash tip and the frictionless flow, $(a-\xi)$ becomes the extent of the swash tip region in the $x$-direction. The flow behind $\xi(t)$ is frictionless and follows the swash solution, Eq. (2.3).

Treating the swash tip in an integral sense (cf. Brocchini and Peregrine, 1996; Archetti and Brocchini, 2002; Brocchini, 2006), the conservation of mass is written as

$$
\frac{\mathrm{d}}{\mathrm{d} t} \int_{\xi(t)}^{a(t)} \rho h(x, t) \mathrm{d} x=\rho h(\xi(t), t)\left[u(\xi(t), t)-\frac{\mathrm{d} \xi(t)}{\mathrm{d} t}\right],
$$

which can be integrated in time using Eq. (2.3) to express $h(\xi(t), t)$ and $\xi(t)$ in terms of $u(\xi(t), t)$ :

$$
\begin{aligned}
\xi(t) & =\frac{3}{2} u(\xi(t), t) t-\frac{1}{2} U_{s} t+g(\sin \theta) t^{2}, \\
h(\xi(t), t) & =\frac{1}{4 g}\left[U_{s}-u(\xi(t), t)-g(\sin \theta) t\right]^{2} .
\end{aligned}
$$

Inserting Eq. (2.5)-(2.6) in Eq. (2.4), and using the initial condition $\left[\int_{\xi(t)}^{a(t)} \rho h(x, t) \mathrm{d} x\right]=0$ at $t=0$, gives

$$
\left[\int_{\xi(t)}^{a(t)} \rho h(x, t) \mathrm{d} x\right]=\frac{\rho t}{8 g}\left[U_{s}-g(\sin \theta) t-u(\xi(t), t)\right]^{3} .
$$

The integral momentum equation for the swash tip is written as

$$
\frac{\mathrm{d}}{\mathrm{d} t} \int_{\xi(t)}^{a(t)} \rho h(x, t) u(x, t) \mathrm{d} x=\rho h(\xi(t), t) u(\xi(t), t)\left[u(\xi(t), t)-\frac{\mathrm{d} \xi(t)}{\mathrm{d} t}\right]+F
$$

where $F$ is the resultant force on the swash tip in the $x$-direction. This resultant force is given by

$$
F=\frac{1}{2} \rho g h^{2}(\xi(t), t)-g \sin \theta \int_{\xi(t)}^{a(t)} \rho h(x, t) \mathrm{d} x-F_{\text {friction }},
$$

where the terms on the right hand side are due to the pressure force from the frictionless flow behind the swash tip, the gravitational deceleration of the swash tip, and the force due to friction 
with the bed, respectively. We do not consider the effects of surface tension at the leading edge of the swash tip, which can be important in small-scale laboratory experiments (see, e.g., Mahony and Pritchard, 1980).

The Pohlhausen method requires that we assume a form of variation for the velocity within the swash tip and we introduce an approximation originally presented by Whitham (1955), which was shown to be correct to the leading order by Hogg and Pritchard (2004): the velocity in the swash tip does not vary with $x$ and is only a function of time. This velocity of the swash tip is denoted as $U(t)$, which is now also the velocity of the shoreline, i.e., $\mathrm{d} a / \mathrm{d} t=U(t)$, and the velocity at the interface, i.e. $u(\xi(t), t)=U(t)$. The force due to friction with the bed is modelled using a quadratic dependency on the swash tip velocity as

$$
\rho K U^{2}(a-\xi)
$$

where $K$ is a dimensionless constant from the parameterization of the bed shear stress, $K=\tau_{b} / \rho U^{2}$. Adopting the same notation as Whitham (1955), the time-varying mass of the swash tip, given by Eq. (2.7), is denoted as $M(t)$. The time-varying momentum of the swash tip is denoted as $P(t)$. The thickness of the water layer at the inferface, $h(\xi(t), t)$, is denoted as $H(t)$. Under the approximation introduced above, the left hand side of the momentum equation, Eq. (2.8), is simply

$$
\frac{\mathrm{d} P}{\mathrm{~d} t}=M \frac{\mathrm{d} U}{\mathrm{~d} t}+U \frac{\mathrm{d} M}{\mathrm{~d} t}
$$

and the mass equation, Eq. (2.4), simplifies to

$$
\frac{d M}{d t}=\rho H\left(U-\frac{d \xi}{d t}\right)
$$

Substituting the above expressions and the modelled term for the bed friction into the momentum equation for the swash tip, Eq. (2.8), gives

$$
M \frac{d U}{d t}=\frac{1}{2} \rho g H^{2}-\rho K U^{2}(a-\xi)-M g \sin \theta .
$$

Inserting the expressions for $\xi, H$, and $M$ in terms of $U$ from (2.5)-(2.7) into Eq. (2.9) and rewritting the equation in terms of $a, d a / d t \equiv U$, and $d^{2} a / d t^{2} \equiv d U / d t$ gives a single equation for the shoreline motion, $a(t)$ :

$$
\begin{aligned}
4 t\left[U_{s}-g(\sin \theta) t-\frac{d a}{d t}\right]^{3}\left(\frac{d^{2} a}{d t^{2}}+g \sin \theta\right)= & {\left[U_{s}-g(\sin \theta) t-\frac{d a}{d t}\right]^{4}-32 K g\left(\frac{d a}{d t}\right)^{2} } \\
& \times\left[a-\frac{3}{2} \frac{d a}{d t} t+\frac{1}{2} U_{s} t-g(\sin \theta) t^{2}\right]
\end{aligned}
$$

When friction is neglected, i.e., $K=0$, the frictionless shoreline solution, Eq. (2.2), can be recovered from the equation above. To make the problem more tractable in the case where friction is considered, we introduce the dimensionless position of the swash tip, $\alpha$, and the dimensionless time, $\tau$,

$$
\alpha=\frac{K g}{U_{s}^{2}}\left[U_{s} t-\frac{1}{2} g(\sin \theta) t^{2}-a\right], \tau=\frac{K g}{U_{s}} t
$$


where $\alpha$ is proportional to the distance between the frictionless shoreline solution, $x_{s}$, and the actual shoreline, $a(t)$. The form of $\alpha$ and $\tau$ are similar to those for the dam-break flow (Whitham, 1955), but modified to suit the swash uprush on a sloping bed. In terms of the dimensionless variables, $d \alpha / d \tau=1-\left[g(\sin \theta) t / U_{s}\right]-\left(1 / U_{s}\right) d a / d t$ and $d^{2} \alpha / d \tau^{2}=-\sin \theta / K-(1 / K g) d^{2} a / d t^{2}$. The dimensionless momentum equation can be derived from Eq. (2.10) as

$$
4 \tau \dot{\alpha}^{3} \ddot{\alpha}+\dot{\alpha}^{4}=16\left[1-\dot{\alpha}-\left(\frac{\sin \theta}{K}\right) \tau\right]^{2}(3 \tau \dot{\alpha}-2 \alpha),
$$

where the "dot" notation represents differentiation with respect to $\tau$, so that $\dot{\alpha} \equiv d \alpha / d \tau$ and $\ddot{\alpha} \equiv d^{2} \alpha / d \tau^{2}$. The solution to this equation is presented in the following section.

\section{Results}

\subsection{Solution for shoreline motion}

Following Whitham (1955), we introduce the parameters $p=\dot{\alpha}$ and $\tau=f^{\prime}(p)$ to transform the dimensionless momentum equation so that $\dot{\alpha}$ becomes the independent variable and $\tau$ becomes the dependent variable. Note, the prime notation, ",", denotes differentiation of $f$. Under this transformation, $\alpha=\int \dot{\alpha} d \tau$, which with the initial condition $\alpha(0)=0$, leads to $\alpha=p f^{\prime}(p)-f(p)$ and $\ddot{\alpha}=d \dot{\alpha} / d \tau=1 / f^{\prime \prime}(p)$. The momentum equation, Eq. (2.11), can be re-written as

$$
4 f^{\prime} p^{3}+p^{4} f^{\prime \prime}=16 f^{\prime \prime}\left[1-p-\left(\frac{s}{K}\right) f^{\prime}\right]^{2}\left(f^{\prime} p+2 f\right) .
$$

At small time, near the initial shoreline position, $p=\dot{\alpha}$ is small and the function $f(p)$ can be represented as a Taylor series expansion about $p=0$ to give

$$
f(p)=f(0)+f^{\prime}(0) p+\frac{1}{2} f^{\prime \prime}(0) p^{2}+\frac{1}{6} f^{(3)}(0) p^{3}+\frac{1}{24} f^{(4)}(0) p^{4}+\ldots
$$

From the initial conditions, at $\tau=0, \alpha=\dot{\alpha}=0$, we obtain $f(0)=f^{\prime}(0)=0$. The coefficients of higher order terms in the Taylor series expansion can be found by substituting Eq. (3.2) into Eq. (3.1), which reveals that $f^{\prime \prime}(0)=0$ and $f^{(3)}(0)=0$. Thus, the leading term in Taylor series expansion of $f(p)$ is proportional to $p^{4}$ and an explicit solution for the shoreline motion, valid at small time, that includes the leading order effect of friction is given by

$$
f^{\prime}(p)=\frac{7}{72} p^{3}+\mathcal{O}\left(p^{4}\right)
$$

which can be re-expressed in the original variables to give

$$
\frac{d a}{d t}=U_{s}-g(\sin \theta) t-\left(\frac{72}{7} U_{s}^{2} K g t\right)^{1 / 3},
$$

where the last term represents the correction due to friction. This Taylor series solution is valid for small times and is equivalent to Whitham's (1955) corresponding small time solution, except for the effect of the slope on the shoreline motion. The above solution can be integrated with a prescribed initial condition $a(0)=0$ to give the shoreline position, also valid at small time, as

$$
a(t)=U_{s} t-\frac{1}{2} g(\sin \theta) t^{2}-\frac{3}{4} t^{4 / 3}\left(\frac{72}{7} U_{s}^{2} K g\right)^{1 / 3} .
$$




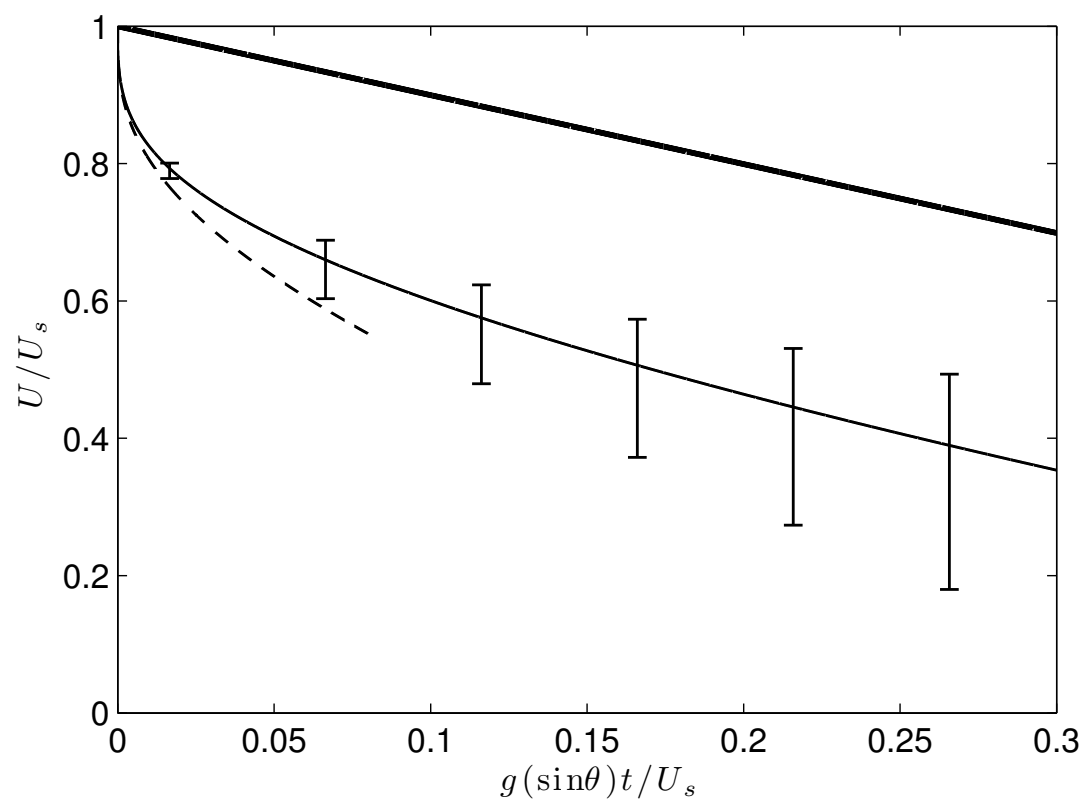

Figure 2: Dimensionless shoreline velocity. —— frictionless solution, Eq. (2.2) with $\tan \theta=1 / 12$; $\longrightarrow$, full numerical solution of Eq. (2.11), with $\tan \theta=1 / 12$ and $K=0.005$; - - Taylor series approximate solution, Eq. (3.4), with $\tan \theta=1 / 12$ and $K=0.005$. Vertical bars show the bounds for the full numerical solution, Eq. (2.11), for $0.0025 \leq K \leq 0.01$.

The Taylor series solution for the shoreline velocity, Eq. (3.3), is re-expressed back in terms of $\alpha$ and $\tau$ to give initial conditions for a numerical solution to Eq. (2.11), which is computed using a fourth-order Runge-Kutta algorithm. The shoreline velocity from the full numeical solution, the Taylor series solution and the frictionless solution are plotted together in figure 2 for the parameters $\tan \theta=1 / 12$ and $K=0.005$. As expected, the Taylor series solution matches the full numerical solution at small times. Both the Taylor series solution and the full numerical solution show an immediate departure from the frictionless solution.

In the following, we discuss the results of the theory for small times and for large times. The theory predicts infinite deceleration of the shoreline due to friction at $t=0$; it was found that $f^{\prime \prime}(0)=0$, which means that $\ddot{\alpha} \rightarrow \infty$ and $d^{2} a / d t^{2} \rightarrow-\infty$ at $t=0$. The same feature can be seen in figure 3. The figure plots the acceleration of the swash tip and the acceleration due to different terms in the momentum budget that are on the right hand side of Eq. (2.9): the onshore-directed acceleration due to the pressure force of the frictionless flow behind the swash tip, the deceleration of the swash tip due to friction, and the deceleration of the swash tip due to gravity. Dividing all terms in Eq. (2.9) by the mass of the swash tip, $M$, it can be seen that the first two terms on the right hand side of the equation may become infinitely large at $t=0$ when the mass of the swash tip vanishes. Using Eq. (3.4)-(3.5), the relative magnitude of these terms at small times can be shown to be

$$
\frac{\rho K U^{2}(a-\xi) / M g}{\rho H^{2} / 2 M} \approx \frac{14}{9}-\frac{32}{(72 / 7)^{2 / 3}}\left(\frac{K g t}{U_{s}}\right)^{1 / 3},
$$

revealing that the deceleration due to friction is greater than the acceleration due to the pressure force of the fluid behind the swash tip as $t \rightarrow 0$. For $t>0$, the swash tip deceleration decreases in magnitude until, at $g(\sin \theta) t / U_{s}=0.33$, it reaches the state where it is equal to the deceleration 


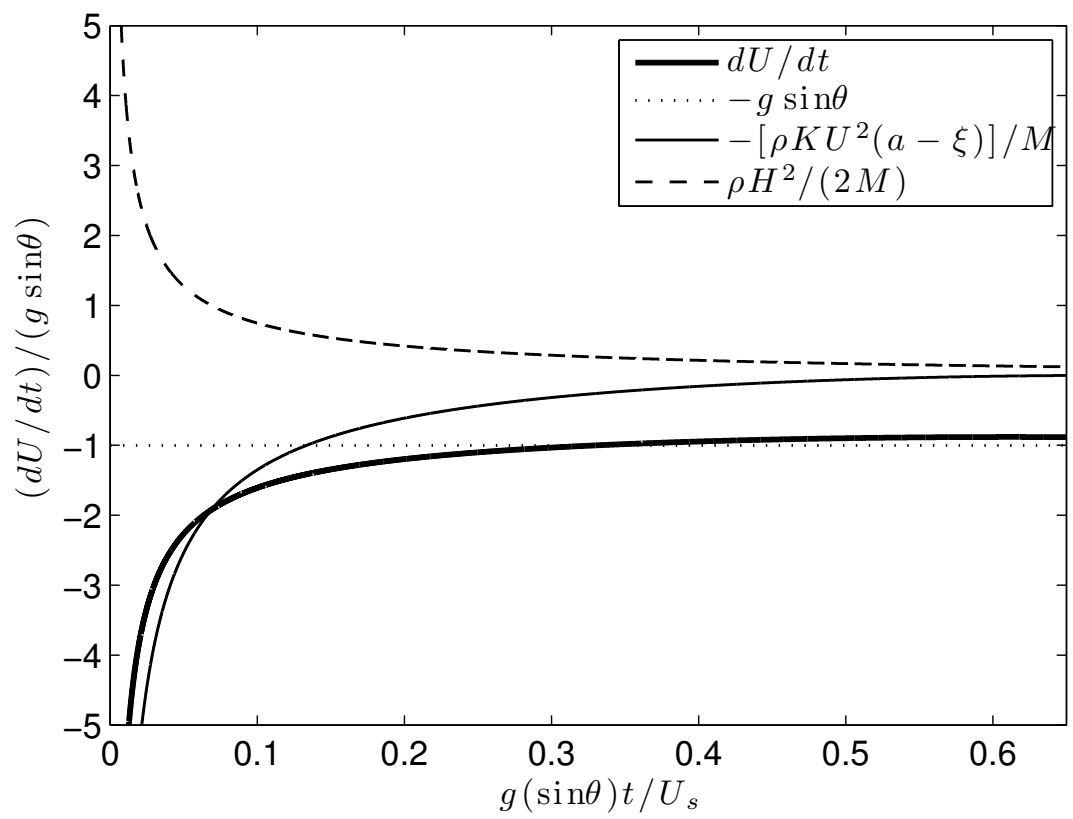

Figure 3: Dimensionless shoreline acceleration and budget. —_, shoreline deceleration from full numerical solution of Eq. (2.11) with $\tan \theta=1 / 12$ and $K=0.005 ; \cdots$, contribution to shoreline deceleration due to gravity; __ , contribution to shoreline deceleration from friction; _ _ , contribution to shoreline acceleration due to the pressure force of the frictionless flow behind the swash tip.

due to gravity alone, i.e., $d U / d t=-g \sin \theta$. At that time, there is a momentary balance between the onshore-directed acceleration due to the force of the frictionless flow behind the swash tip and the offshore-directed deceleration due to bed friction. From Eq. (2.6), it can also be shown that the water depth at the interface, $H$, reaches its maximum value when $d U / d t=-g \sin \theta$.

For the same parameters $(\tan \theta=1 / 12, K=0.005)$, the dimensionless shoreline position, $g(\sin \theta) a / U_{s}^{2}$, and the dimensionless position of the interface, $g(\sin \theta) \xi / U_{s}^{2}$, are plotted together in figure 4. The dimensionless extent of the swash tip, $g \sin \theta(a-\xi) / U_{s}^{2}$, is initially zero and increases throughout the uprush. The approximation made for the Pohlahusen method, i.e., the velocity within the swash tip does not vary with $x$, becomes invalid when $(a-\xi)$ becomes large and the results of the theory may not be accurate thereafter. The precise time after which the theory no longer yields accurate results is not known since measurements of velocity in the swash tip are not available, but it will depend on values of the beach slope, $\theta$, and the dimensionless constant, $K$, since the parameter $(\sin \theta / K)$ appears in Eq. (2.11). Beyond the state where $d U / d t=-g \sin \theta$, which occurs at $g(\sin \theta) t / U_{s}=0.33$ for the parameters $\tan \theta=1 / 12$ and $K=0.005$, the shoreline deceleration is predicted to be very close to that due to gravity alone for the remainder of the uprush (see figure 3). In other words, the results of the theory predict that friction is negligible in the later stages of the uprush. Laboratory and field observations show that the shoreline decelerates due to friction throughout the uprush and thus, we may say that the predictions of shoreline motion from the theory for $g(\sin \theta) t / U_{s}>0.33$ are not accurate for the values of $\theta$ and $K$ shown here. 


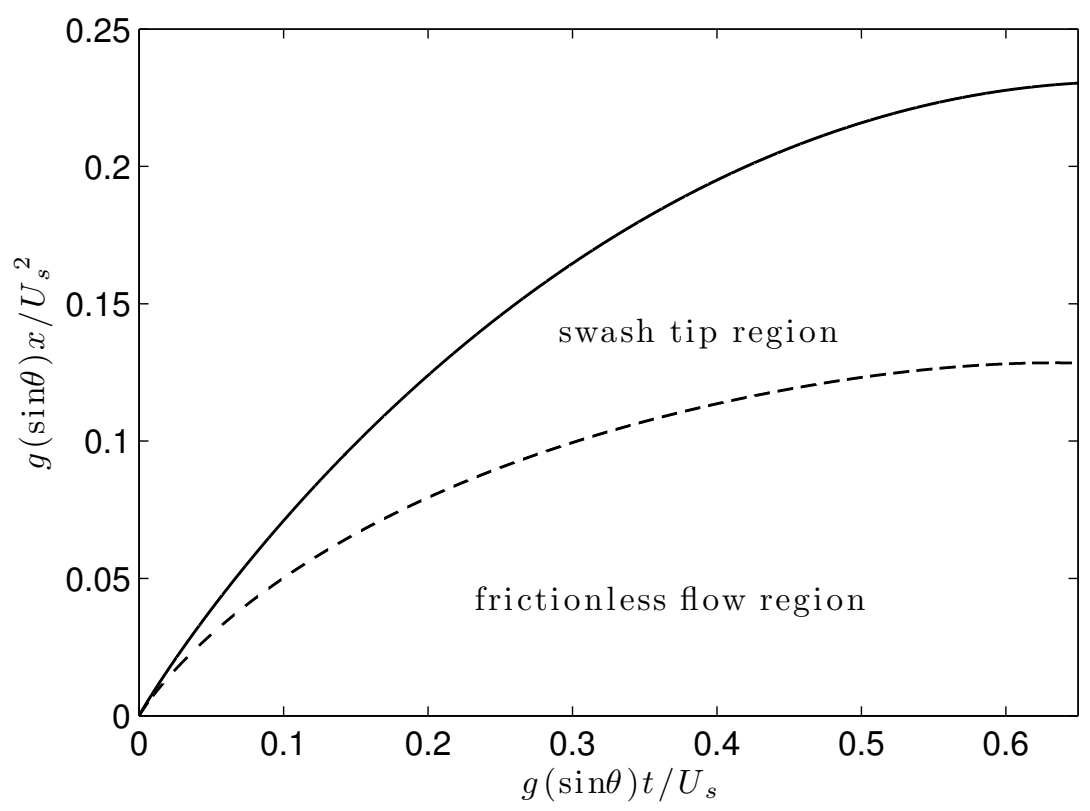

Figure 4: Dimensionless position of the shoreline and the interface between frictionless flow and the swash tip. — full numerical solution of Eq. (2.11), $x=a(t)$, with $\tan \theta=1 / 12$ and $K=0.005$; - - , position of the interface, $x=\xi(t)$, calculated using the full numerical solution and Eq. (2.5) for $\tan \theta=1 / 12$ and $K=0.005$.
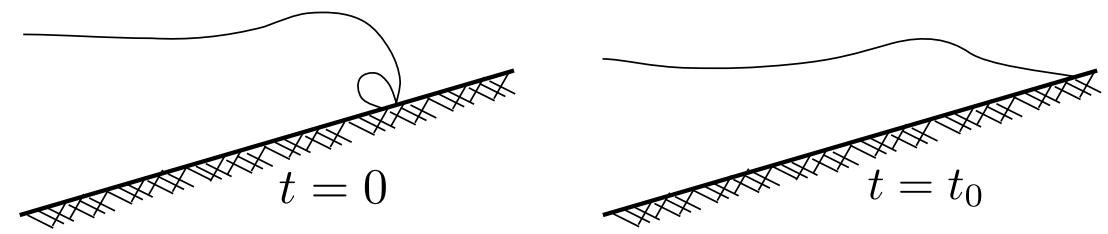

Figure 5: Sketches of plunging breakers in Pujara et al. (2015) near the stillwater shoreline. Left: $t=0$ is the time the overturning jet hits the land onshore of the stillwater shoreline; right: the flow away from the shoreline starts to follow the swash solution, Eq. (2.3), after some short time, $t=t_{0}$. 


\subsection{Comparison to experimental data}

We now compare the results of the theory to measurements of shoreline motion due to breaking solitary waves. Pujara et al. (2015) made measurements of the shoreline velocity and the bed shear stress near the moving shoreline for a range of different breaking solitary waves on an impermeable sloping beach with $\tan \theta=1 / 12$. In the experiments, $t=0$ was defined as the moment the shoreline begins to move when the overturning crest of the plunging breaker crashes onto the beach, as shown in the left sketch of figure 5. However, unlike the predictions of shallow water theory, the shoreline accelerated for a short duration, $t \leq t_{0}$, until the collapse of the waveform was completed. Shoreline accelerations at or near $t=0$ have been previously observed in laboratory experiements (Yeh et al., 1989) and numerical computations (Hibberd and Peregrine, 1979) and it is known that the shallow water theory breaks down during the bore collapse process (Whitham, 1958). The formulation of the theory in the previous section assumes a shallow water framework where bore collapse occurs instantaneously at $t=0$ leading to the swash solution, Eq. (2.3), for $t>0$, whereas in reality, the flow will only be well-approximated by the shallow water theory and hence follow the swash solution after the bore collapse process is completed. In $(x, t)$-space, the shallow water theory can be said to apply for $(x, t)>\left(x_{0}, t_{0}\right)$ for some small values of $x_{0}$ and $t_{0}$ (Peregrine and Williams, 2001). The right sketch of figure 5 depicts the situtaion at $t=t_{0}$. As previously mentioned, the theory developed in the preceeding section for shoreline motion predicts infinite deceleration of the shoreline at $t=0$. Since the theory for shoreline motion assumes a shallow water framework for all times, the deceleration predicted by the theory during $0 \leq t \leq t_{0}$ can be said to be unphysical. The loss of shoreline velocity incurred during this time becomes problematic when comparing results of the theory to experimental data. To adequately compare the results of the theory to the experimental data, a constant velocity is added to the the results of the theory that compensate for the unphysical deceleration during $0 \leq t \leq t_{0}$. This constant, denoted $\Delta U_{s}$, is calculated as the loss of shoreline velocity during $0 \leq t \leq t_{0}$ due to the the frictional term in Taylor series solution, i.e. the last term on the right hand side of Eq. (3.4) with $t$ replaced by $t_{0}$. In dimensionelss form, it is give by

$$
\frac{\left(\Delta U_{s}\right)}{U_{s}}=\left(\frac{72}{7} \frac{K g t_{0}}{U_{s}}\right)^{1 / 3} .
$$

Figure 6 plots the data of shoreline velocity of four plunging breakers (W7-10 in Pujara et al., 2015) made dimensionless by their measured initial shoreline velocity, $U_{s}$. The data in figure 6 show that the dimensionless shoreline velocity of all four plunging breakers collapse onto each other for the early stages of the uprush suggesting that a single dimensionless solution for the friction-affected shoreline motion is reasonable. This hypothesis is further supported by Pujara et al.'s bed shear stress data at $g x / U_{s}^{2} \approx 0.1$ that shows that $K=\tau_{b} / \rho U_{s}^{2} \approx 0.005$ at the swash tip for all four plunging breakers. Figure 6 shows the comparison between the full numerical solution of Eq. (2.11) with $\tan \theta=1 / 12$ and $K=0.005$ and the shoreline velocity data for all four plunging breakers. The results of the theory are compensated by adding a constant, $\Delta U_{s} / U_{s}=0.20$, which is calculated using Eq. (3.7) with $g t_{0} / U_{s}=0.15$. In the data, the time at which the shoreline velocities for different breakers reach their maximum value (indicating that the collapse is complete) falls in the range $0.11 \leq g t_{0} / U_{s} \leq 0.21$, with $g t_{0} / U_{s}=0.15$ being the average value. The constant calculated using Eq. (3.7) falls in the range $0.18 \leq \Delta U_{s} / U_{s} \leq 0.22$ and so the comparison in figure 6 is not sensitive to its exact value. It can be seen that the results from the theory match the data very well for the early stages of the uprush, which is when the data also collapse and a single dimensionless solution for shoreline motion of different breakers is possible. It should be noted 


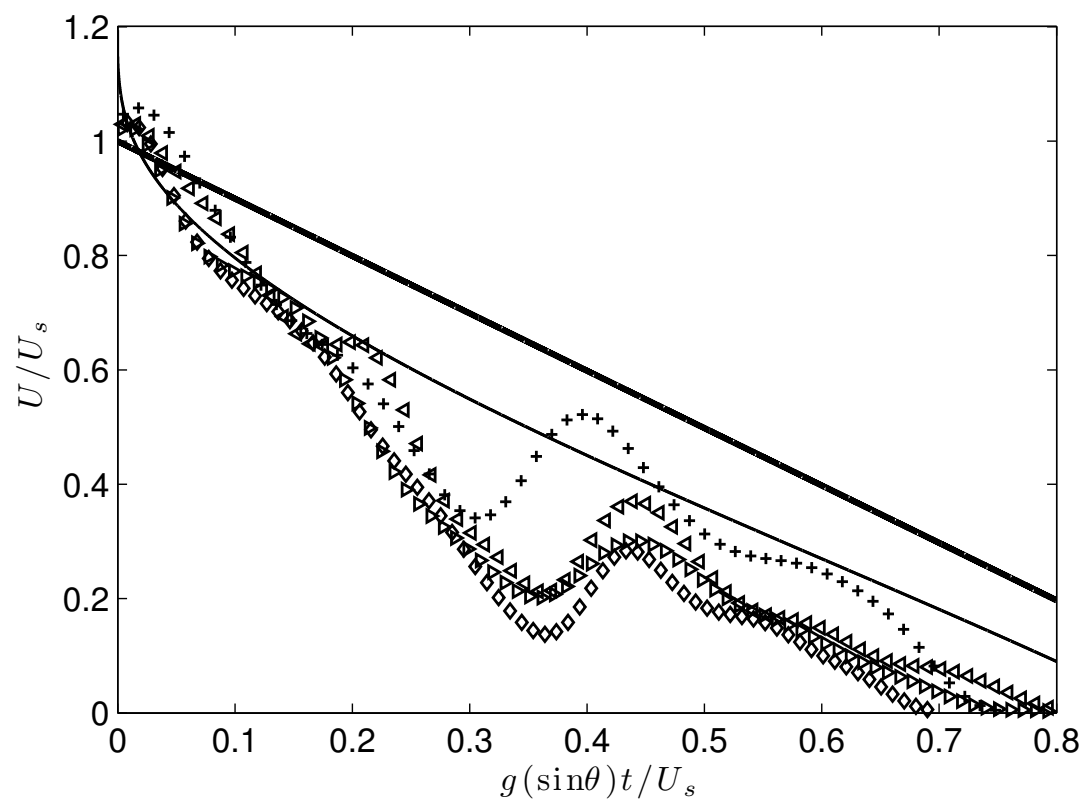

Figure 6: Dimensionless shoreline velocity, comparison of experimental data (Pujara et al., 2015) to theoretical solutions. +, W7 data; $\triangleleft$, W8 data; $\triangleright$, W9 data; $\diamond$, W10 data; — , frictionless solution, Eq. (2.2), with $\tan \theta=1 / 12$; —_, full numerical solution, Eq. (2.11), with $\tan \theta=1 / 12$ and $K=0.005$. A constant, $\Delta U_{s} / U_{s}=0.20$, is added to the full numerical solution.

that this match is achieved using a constant value of $K$ that is not fitted to the shoreline motion data. Small changes in the choice for the value of $K$ would not significantly alter the results as seen in figure 2, where vertical bars show the solution for a factor of two change in the value of $K$. Knowledge of the variation in the value of $K$ for different laboratory and field conditions would be useful to to aid further application of these results. The data in figure 6 also show an oscillatory signal superimposed onto the shoreline deceleration, which may be caused by larger scale motions created during wave breaking.

The theory assumes that a constant value of $K$ is able to correctly parameterize the bed shear stress throughout the uprush, but the bed shear stress data from Pujara et al. (2015) do not support this assumption (see also e.g. Barnes et al., 2009; Barnes and Baldock, 2010). Thus, the agreement between experimental data and theory deteriorates for $g(\sin \theta) t / U_{s}>0.2$. In any case, we expect the theory to break down during later stages of uprush, as discussed in the previous section and seen in the results from the theory in figure 6 . In the time range $0.35 \leq g t / U_{s} \leq 0.45$, the experimental data show shoreline accelerations, which has been linked to a 'mini-collapse' caused by a build up of fluid behind the shoreline as it comes to a halt (see Yeh et al., 1989; Zhang and Liu, 2008). This feature is not captured by the theory, of course.

Measurements of the shoreline position from the experiments made dimensionless by $U_{s}^{2} / g \sin \theta$ are plotted in figure 7 and compared to the theoretical solutions. Since the overturning jet of the plunging breakers crashed onshore of the stillwater shoreline, the data for shoreline position do not begin at $x=0$. In the experiments, the average starting position for the plunging breakers was $g(\sin \theta) x / U_{s}^{2}=0.024$ and the theoretical solutions are also initialized at this value. Similar to shoreline velocity, the theoretical solutions provide an encouraging match to the experimental data for $g(\sin \theta) t / U_{s} \leq 0.2$, after which there is some deviation between the data of different waves and the full numerical solution. 


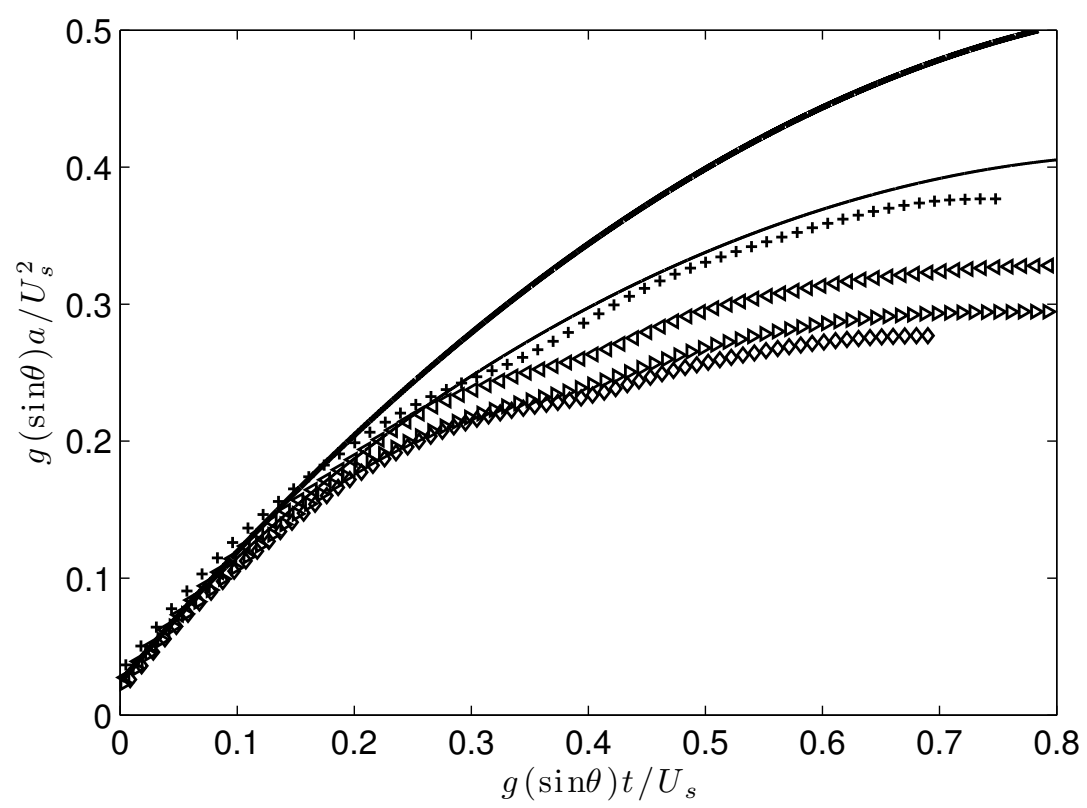

Figure 7: Dimensionless shoreline position, comparison of experimental data (Pujara et al., 2015) to theoretical solutions. +, W7 data; $\triangleleft$, W8 data; $\triangleright$, W9 data; $\diamond$, W10 data; - , frictionless solution, Eq. (2.2), with $\tan \theta=1 / 12$; — , full numerical solution, Eq. (2.11), with $\tan \theta=1 / 12$ and $K=0.005$. A constant, $g(\sin \theta) x / U_{s}^{2}=0.024$, is added to the full numerical solution to reflect the average starting position for the plunging breakers in the experimental data.

Finally, we consider the predictions of the run-up from the theoretical solution, which are the first predictions of run-up of breaking solitary waves not based on an empirical fit to previous run-up data, though they do include empirical parameters. The run-up, $R$, is presented in Table 1 from three sources: (i) experimental data, $R_{\text {data }}$; (ii) predictions of full numerical solution to Eq. (2.11), $R_{\text {num }}$; (iii) predictions from the frictionless shoreline solution, Eq. (2.2), $U_{s}^{2} / 2 g$. The full numerical solution generally over-predicts the run-up, but provides a significant improvement to the predictions of the frictionless solution and can serve as an improved upper-bound on the experimentally measured run-up.

\begin{tabular}{lcccccc}
\hline Wave & $h_{0}(\mathrm{~m})$ & $H_{0}(\mathrm{~m})$ & $U_{s}(\mathrm{~m} / \mathrm{s})$ & $R_{\text {data }}(\mathrm{m})$ & $R_{\text {num }}(\mathrm{m})$ & $U_{s}^{2} / 2 g(\mathrm{~m})$ \\
\hline W7 & 1.72 & 0.345 & 5.0 & 0.62 & 1.05 & 1.27 \\
W8 & 1.72 & 0.410 & 5.7 & 0.81 & 1.36 & 1.66 \\
W9 & 1.72 & 0.443 & 6.2 & 0.96 & 1.61 & 1.96 \\
W10 & 1.72 & 0.493 & 6.6 & 1.08 & 1.83 & 2.22 \\
\hline
\end{tabular}

Table 1: Comparison of run-up predictions to data from Pujara et al. (2015) for breaking solitary waves. $h_{0}$ is the stillwater depth in the flat bed region, $H_{0}$ is the wave height in the flat bed region, and the slope of the plane beach at the end of the flat bed region is $\tan \theta=1 / 12 . U_{s}$ is the measured initial shoreline velocity. The value of the friction coefficient used in the calculations of run-up from the theoretical solutions is $K=0.005$. 


\section{Conclusion}

A theory has been developed that provides an integral treatment of the swash tip, including the effects of bed friction, during the swash uprush by extending the 'Pohlhausen method' used by Whitham (1955). With the use of the simple model - the bed shear stress being proportional to the square of the flow speed - the swash tip is modelled as a friction-affected region and the flow evolution behind the swash tip is given by the Peregrine and Williams (2001) swash solution, Eq. (2.3). The theory is used to obtain shoreline velocities and positions, which are compared to laboratory data of shoreline motion due to breaking solitary waves. The theory demonstrates the ability to capture the leading order dynamical balance at the moving shoreline during the initial stages of the uprush, which is when the deceleration of the shoreline is most affected by friction. The theory also provides predictions of the run-up of breaking solitary waves that show a better agreement with experimental data than the frictionless solution.

\section{Acknowledgements}

The authors gratefully acknowledge the support of the National Science Foundation (CMMI1041541) and the help of M. Park, T. Maddux, J. Killian and A. Ryan at the Hinsdale Wave Research Laboratory at Oregon State University, for their help with experimental setup and data collection for the data presented herein. The help of Y. S. Park during the initial design stages of the shear plate sensor, and the help P. Charles, T. Brock, J. Powers and C. Willkens at Cornell University with the construction of the shear plate sensor is also acknowledged. We also thank two reviewers, whose comments helped to improve the manuscript. 


\section{References}

C Ancey, R M Iverson, M Rentschler, and R P Denlinger. An exact solution for ideal dam-break floods on steep slopes. Water Resources Research, 44:W01430, 2008.

M Antuono. A shock solution for the nonlinear shallow water equations. Journal of Fluid Mechanics, 658:166-187, 2010.

M Antuono and A J Hogg. Run-up and backwash bore formation from dam-break flow on an inclined plane. Journal of Fluid Mechanics, 640:151-164, 2009.

M Antuono, L Soldini, and M Brocchini. On the role of the Chezy frictional term near the shoreline. Theoretical and Computational Fluid Dynamics, 26:105-116, 2012.

$\mathrm{R}$ Archetti and M Brocchini. An integral swash zone model with friction: an experimental and numerical investigation. Coastal Engineering, 45:89-110, 2002.

T. E. Baldock, D. Peiris, and A. J. Hogg. Overtopping of solitary waves and solitary bores on a plane beach. Proceedings of the Royal Society A, 468:3494-3516, 2012.

T E Baldock, R Grayson, B Torr, and H E Power. Flow convergence at the tip and edges of a viscous swash front — Experimental and analytical modeling. Coastal Engineering, 88:123-130, 2014.

M P Barnes and T E Baldock. A Lagrangian model for boundary layer growth and bed shear stress in the swash zone. Coastal Engineering, 57:385-396, 2010.

M P Barnes, T O'Donoghue, J M Alsina, and T E Baldock. Direct bed shear stress measurements in bore-driven swash. Coastal Engineering, 56:853-867, 2009.

AGL Borthwick, M Ford, B P Weston, P H Taylor, and P K Stansby. Solitary wave transformation, breaking and run-up at a beach. Proceedings of the ICE-Maritime Engineering, 159:97-105, 2006.

M Brocchini. Integral swash-zone models. Continental Shelf Research, 26:653-660, 2006.

M. Brocchini and T. E. Baldock. Recent advances in modeling swash zone dynamics: Influence of surf-swash interaction on nearshore hydrodynamics and morphodynamics. Reviews of Geophysics, 46:RG3003, 2008.

M Brocchini and D H Peregrine. Integral flow properties of the swash zone and averaging. Journal of Fluid Mechanics, 317:241-273, 1996.

$\mathrm{H}$ Chanson. Application of the method of characteristics to the dam break wave problem. Journal of Hydraulic Reseach, 47:41-49, 2009.

E. A. Cowen, I. M. Sou, P. L.-F. Liu, and B. Raubenheimer. Particle image velocimetry measurements within a laboratory-generated swash zone. Journal of Engineering Mechanics, 129: 1119-1129, 2003.

S. Hibberd and D. H. Peregrine. Surf and run-up on a beach: a uniform bore. Journal of Fluid Mechanics, 95:323-345, 1979. 
A J Hogg and D Pritchard. The effects of hydraulic resistance on dam-break and other shallow inertial flows. Journal of Fluid Mechanics, 501:179-212, 2004.

M G Hughes. Friction factors for wave uprush. Journal of Coastal Reseach, 11:1089-1098, 1995.

A. Jensen, G. K. Pedersen, and D. J. Wood. An experimental study of wave run-up at a steep beach. Journal of Fluid Mechanics, 468:161-188, 2003.

M S Kirkgöz. A theoretical study of plunging breakers and their run-up. Coastal Engineering, 5: $353-370,1981$.

P. Lin, K.-A. Chang, and P. L.-F. Liu. Runup and rundown of solitary waves on sloping beaches. Journal of Waterway, Port, Coastal, and Ocean Engineering, 125:247-255, 1999.

P J Lynett, T.-R. Wu, and P. L.-F. Liu. Modeling wave runup with depth-integrated equations. Coastal Engineering, 46:89-107, 2002.

J. J. Mahony and W. G. Pritchard. Wave reflexion from beaches. Journal of Fluid Mechanics, 101:809-832, 1980.

R E Meyer and A D Taylor. Waves on beaches and resulting sediment transport, chapter Run-up on beaches, pages 357-411. Academic Press New York, 1972.

A R Packwood and D H Peregrine. Surf and run-up on beaches: models of viscous effects. Technical Report AM-81-07, University of Bristol, Bristol, UK, 1981.

G K Pedersen, E LindstroǏžm, A F Bertelsen, A Jensen, D Laskovski, and G Sælevik. Runup and boundary layers on sloping beaches. Physics of Fluids, 25:012102, 2013.

D H Peregrine. Waves on beaches and resulting sediment transport, chapter Equations for water waves and the approximations behind them, pages 95-121. Academic Press, New York, 1972.

D H Peregrine and S M Williams. Swash overtopping a truncated plane beach. Journal of Fluid Mechanics, 440:391-399, 2001.

N Pujara, P L-F Liu, and H Yeh. The swash of solitary waves: flow evolution, bed shear stress and run-up. Journal of Fluid Mechanics, 779:556-597, 2015.

J A Puleo and K T Holland. Estimating swash zone friction coefficients on a sandy beach. Coastal Engineering, 43:25-40, 2001.

M C Shen and R E Meyer. Climb of a bore on a beach. Part 3. Run-up. Journal of Fluid Mechanics, 16:113-125, 1963.

I. M. Sou and H. Yeh. Laboratory study of the cross-shore flow structure in the surf and swash zones. Journal of Geophysical Reseach, 116:C03002, 2011.

I A Svendsen. Introduction to Nearshore Hydrodynamics, volume 24 of Advanced Series on Ocean Engineering. World scientific, 2006.

G B Whitham. The effects of hydraulic resistance in the dam-break problem. Proceedings of the Royal Society A, 227:399-407, 1955. 
G B Whitham. On the propagation of shock waves through regions of non-uniform area or flow. Journal of Fluid Mechanics, 4:337-360, 1958.

H H Yeh, A Ghazali, and I Marton. Experimental study of bore run-up. Journal of Fluid Mechanics, 206:563-578, 1989.

Q Zhang and P L-F Liu. A numerical study of swash flows generated by bores. Coastal Engineering, 55:1113-1134, 2008. 